\title{
Atrial Activation During Sinus Rhythm in Patients with Rheumatic and Non-Rheumatic Paroxysmal Atrial Fibrillation. Frequency- Domain Analysis Using Signal-Averaged Electrocardiography
}

\author{
Rogério Carregoza Dantas, Eduardo Corrêa Barbosa, Paulo Roberto Benchimol Barbosa, \\ Alfredo de Souza Bomfim, Paulo Ginefra
}

Rio de Janeiro, RJ - Brazil

\begin{abstract}
Objective - Using P-wave signal-averaged electrocardiography, we assessed the patterns of atrial electrical activation in patients with idiopathic atrial fibrillation as compared with patterns in patients with atrial fibrillation associated with structural heart disease.
\end{abstract}

Methods - Eighty patients with recurrent paroxysmal atrial fibrillation were divided into 3 groups as follows: group I - 40 patients with atrial fibrillation associated with non-rheumatic heart disease; group II - 25 patients with rheumatic atrial fibrillation; and group III - 15 patients with idiopathic atrial fibrillation. All patients underwent $P$-wave signal-averaged electrocardiography for frequency-domain analysis using spectrotemporal mapping and statistical techniques for detecting and quantifying intraatrial conduction disturbances.

Results - We observed an important fragmentation in atrial electrical conduction in $27 \%$ of the patients in group I, $64 \%$ of the patients in group II, and $67 \%$ of the patients in group III $(p=0.003)$.

Conclusion - Idiopathic atrial fibrillation has important intraatrial conduction disturbances. These alterations are similar to those observed in individuals with rheumatic atrial fibrillation, suggesting the existence of some degree of structural involvement of the atrial myocardium that cannot be detected with conventional electrocardiography and echocardiography.

Key words: atrial fibrillation, signal-averaged electrocardiography, idiopathic atrial fibrillation

Universidade do Estado do Rio de Janeiro - UERJ

Mailing address: Paulo Ginefra - Rua Barão de Itambi, 21/702 - 22231-001 - Rio de Janeiro, RJ, Brazil - E-mail: rcdantas@ cardiol.br

English version by Stela Maris C. e Gandour
Atrial fibrillation is the most common sustained arrhythmia diagnosed in clinical practice ${ }^{1-4}$. In the general population, its prevalence increases with age: it is lower than $1 \%$ in the age bracket from 40 to 65 years, ranges from $2 \%$ to $5 \%$ in the age bracket from 65 to 74 years, and is higher than $5 \%$ above the age of 75 years. No difference in incidence has been observed between the sexes ${ }^{1}$. The increase in prevalence and incidence of atrial fibrillation with age is mainly associated with coexisting cardiac diseases. However, an independent effect of direct ageing on the atrial tissue cannot be excluded ${ }^{5}$.

A history of congestive heart failure, rheumatic valvar disease, left ventricular hypertrophy, and left atrial enlargement are independent risk factors associated with the genesis of atrial fibrillation. The presence of this arrhythmia in structurally normal hearts, although infrequent, may be detected in clinical practice. The triggering and maintenance factors of idiopathic atrial fibrillation are not very well defined. However, a significant number of patients with the paroxysmal or persistent form of atrial fibrillation, even though without evidence of anatomical cardiac alterations, evolve towards chronic atrial fibrillation. Such patients include those with structural heart disease despite the use of antiarrhythmic drugs. The objective of our study was to identify the electrophysiological behavior of atrial activation in patients with paroxysmal or persistent atrial fibrillation, through the use of P-wave signal-averaged electrocardiography for frequency-domain analysis.

\section{Methods}

In a cross-sectional study, we analyzed 80 patients who were being followed up in the outpatient cardiac arrhythmia care unit of the Hospital Universitário Pedro Ernesto. These patients had a history of at least 2 episodes of paroxysmal or persistent atrial fibrillation confirmed on electrocardiography in the last year, and they had not used any antiarrhythmic drug for at least 5 half-lives of each drug in 
question. The study was carried out from December 1996 to December 1997. These patients were followed up in the outpatient clinics, assessed through anamnesis, physical examination, electrocardiography, and two-dimensional Doppler echocardiography. They were divided into 3 groups according to the cause of paroxysmal or persistent atrial fibrillation as follows:

Group I - 40 patients with a mean age of $49.2 \pm 15.8$ years, 21 being females, with paroxysmal or persistent atrial fibrillation secondary to different heart diseases as follows: ischemic (16 patients), hypertrophic (7 patients), hypertensive (3 patients), chagasic ( 8 patients), and idiopathic cardiomyopathy (6 patients).

Group II - 25 patients with a mean age of $42.1 \pm 14.3$ years, 11 being females, all of whom had paroxysmal or persistent atrial fibrillation associated with rheumatic involvement of the mitral valve.

Group III - 15 patients with a mean age of $62.9 \pm 18.4$ years, 6 being females, all of whom had idiopathic paroxysmal or persistent atrial fibrillation.

Those patients in whom no data regarding atrial fibrillation could be found in the clinical history, on physical examination, electrocardiogram, echocardiogram, or in the laboratory tests for thyroid function that could correlate with a primary cause were considered as having idiopathic paroxysmal or persistent atrial fibrillation.

After the patients provided written consent to take part in the study, they underwent P-wave signal-averaged electrocardiography. All tests were performed in the morning. The records were obtained withmodel IIc ART-Corazonicsequipment (ARTInc., Texas, USA) and later analyzed by a single observer.

Frank's orthogonal system with 3 bipolar leads and inversion of the $\mathrm{X}$ and $\mathrm{Y}$ axes was used for data acquisition. The $\mathrm{Y}$ vector was positioned on the $4^{\text {th }}$ intercostal space, in the midaxillary line, the negative pole to the right, and the positive pole to the left. The $\mathrm{X}$ vector was positioned with its negative pole in the manubrium and its positive pole in the left costal margin in the midclavicular line.

Signal-averaged electrocardiography was performed by displacing the fiducial point to the right of the coherent averaging window, with total exposure of the P-wave. The correlation window with $30 \mathrm{~ms}$ of duration was positioned on top of the Pwave, and the correlation coefficient was fixed at 0.99 . The noise window was positioned on top of the TP segment and had a duration of $100 \mathrm{~ms}$. The final coherent averaging noise was established in $0.3 \mu \mathrm{V}$. Recognition of the P-wave morphology was specific for the wave used as a reference, and the aberrant atrial complexes were successfully rejected.

After coherent averaging, the frequency-domain analysis of the $\mathrm{P}$ wave was performed using the spectro-temporal mapping technique. The maps were built by dividing the area of analysis upon the $P$ wave into segments of $16 \mathrm{~ms}$ of duration, separated by intervals of $2 \mathrm{~ms}$, multiplied by a tapering window of the Blackman-Harris type. Determination of the content of signal frequency was performed, applying the fast Fourier transform with 512 points for each segment. The signals of each segment were preprocessed for the calculation of the numerical derivative, and then, the arithmetic mean was subtracted. The maps of the $\mathrm{X}, \mathrm{Y}$, and $\mathrm{Z}$ leads were added for building the spectral vectorial magnitude and further analysis.

Based on the spectral map, we analyzed the mean (CEM) and standard deviation (CEDP) of the intersegmentar linear correlation between the amplitudes of the power spectra of the adjacent segments. We also analyzed the standard deviation of the frequency add track (BDDP) of the concentration of energy of the power spectrum of each segment during atrial activation. Intersegmentar correlation analyzes the amplitude distribution of the power spectrum of between consecutive segments throughout atrial activation. Absolutely uniform conduction of the electrical stimulus would be hypothetically represented by a mean equal to 1 and standard deviation equal to zero, indicating perfect correlations between the segments. The values were multiplied by 100 to facilitate reading and manipulation.

The add track, on one side, identifies the concentration of energy corresponding to $80 \%$ of the area of the power spectrum of the segment analyzed in Hertz. The following cut values, already established in previous publications $^{6}$, were considered abnormal: CEM $\leq 78$, DP CEM $\geq 32$, and CEDP $\geq 75 \mathrm{~Hz}$. The examination is considered positive for the presence of fragmented atrial potentials, when 2 or more variables are abnormal.

Data of all variables obtained were filed and analyzed using a personal computer and version 6 of the EPI Info program. The variables of the groups were analyzed using the 2tailed Student $t$ test for 2-by-2 comparisons and 1-way analysis of variance (ANOVA) for comparison of multiple means with normal distribution. The chi-square $\left(\chi^{2}\right)$ test with Yates correction was used for comparing the frequency distributions. The level of alpha significance was fixed at 0.05 . The variables CEDP and BDDP were transformed into their natural logarithms prior to the statistical analysis for normalization of their functions of distribution of probability.

\section{Results}

Gender distribution in the groups was similar, with no statistically significant differences. In regard to age, we observed a higher mean in group III as compared with groups I and II (ANOVA; $\mathrm{p}<0.001$ ).

The means and standard deviations of the variables

\begin{tabular}{|c|c|c|c|c|}
\hline Variable & Group I & Group II & Group III & $\mathrm{p}^{\mathrm{a}}$ \\
\hline CEM & $80.7 \pm 7.8$ & $76.3 \pm 9.3$ & $75.5 \pm 8.1$ & 0.04 \\
\hline CEDP & $28.9 \pm 11.5$ & $33.0 \pm 12.9$ & $35.9 \pm 10.6$ & - \\
\hline CEDP LnT & $3.2 \pm 0.6$ & $3.4 \pm 0.5$ & $3.5 \pm 0.3$ & 0.14 \\
\hline BDDP & $57.4 \pm 12.5$ & $57.2 \pm 12.8$ & $67.1 \pm 13.0$ & - \\
\hline BDDP LnT & $4.0 \pm 0.2$ & $4.0 \pm 0.2 \mathrm{y}$ & $4.2 \pm 0.2$ & 0.04 \\
\hline \multicolumn{5}{|c|}{$\begin{array}{l}\text { CEM- mean of the segmentary linear correlation; CEDP- standard deviation } \\
\text { of the segmentary linear correlation; BDDP- standard deviation of the add } \\
\text { track; ' level of significance of the "one-way" analysis; á p }<0.05 \text { group I vs } \\
\text { group II; İ p }<0.05 \text { group I vs group III; y p }<0.05 \text { group II vs group III. }\end{array}$} \\
\hline
\end{tabular}




\begin{tabular}{|c|c|c|c|c|c|}
\hline \multicolumn{6}{|c|}{$\begin{array}{l}\text { Table II - Incidence of abnormal values of the variables analyzed } \\
\text { on signal-averaged electrocardiogram in the groups studied ( } \% \text { of } \\
\text { abnormal values) }\end{array}$} \\
\hline Variable & Group I & Group II & Group III & $\chi^{2}$ & $\mathrm{p}$ \\
\hline CEM & 32.5 & 64.0 & 66.6 & 8.482 & 0.014 \\
\hline CEDP & 45.0 & 64.0 & 73.3 & 4.446 & 0.108 \\
\hline BDDP & 7.5 & 12.0 & 26.6 & 3.672 & 0.159 \\
\hline Positive test ${ }^{*}$ & 27.5 & 64.0 & 66.6 & 11.34 & 0.003 \\
\hline \multicolumn{6}{|c|}{$\begin{array}{l}\text { CEM - mean of the segmentary linear correlation; CEDP- standard de- } \\
\text { viation of the segmentary linear correlation; BDDP- standard deviation } \\
\text { of the add track; }{ }^{*} \text { defined by the presence of } 2 \text { or more abnormal variables. }\end{array}$} \\
\hline
\end{tabular}

studied are shown in table I. We observed that for the CEM variable, group I had higher values than groups II and III (ANOVA; $p=0.04$ ). The CEDP variable showed no statistically significant difference between the 3 groups. The BDDP index showed statistically significant differences, which were greater in group III as compared with those in groups I and II (ANOVA; $\mathrm{p}=0.04$ ).

Table II shows the incidence of abnormal values of the variables analyzed in the groups studied and the incidence of positive examinations for fragmented atrial potentials.

When looked at individually, only the CEM variable showed a statistically significant difference, which was greater in group I as compared with the other groups. In the analysis of fragmented atrial potentials, groups II and III had a higher incidence of positive examinations than did group I. Figure 1 shows the P-wave signal-averaged electrocardiogram frequency-domain analysis of patients in groups I (negative examination) and III (positive examination).

\section{Discussion}

Slowness in the conduction of the electrical stimulus and dispersion of atrial refractoriness constitute the major factors in the formation of reentrant circuits in the atria, especially in compromised hearts ${ }^{7}$. Structural changes in the atrial tissue are also related to factors predisposing one to this heterogeneity in electrical conduction and excitation ${ }^{8}$. Age, atrial distension, interaction with the autonomous

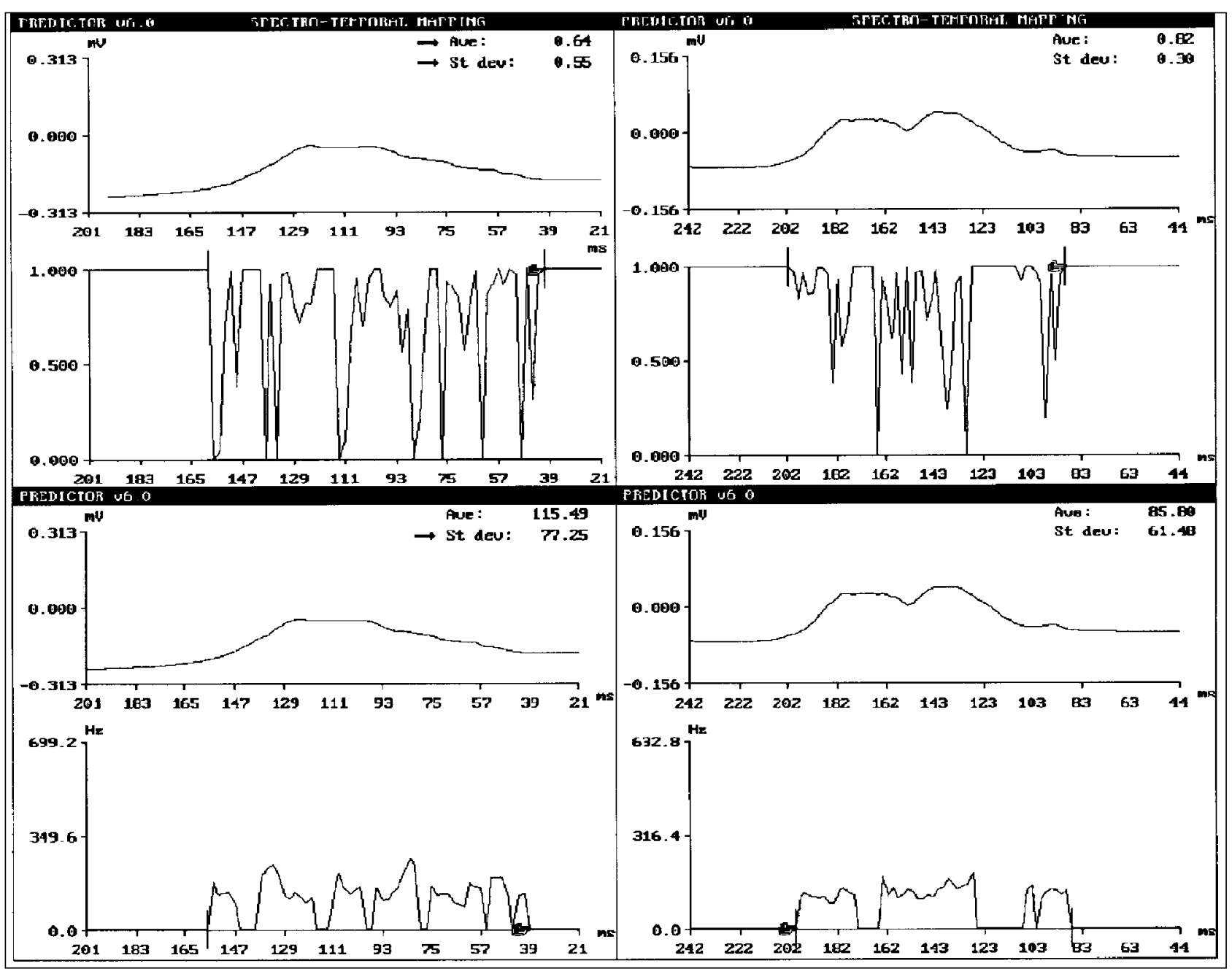

Fig. 1 - P-wave signal-averaged electrocardiography for frequency-domain analysis of the patients in groups I and III. On top, the spectral correlation technique, and at the bottom, the frequency add track. To the left, in a patient with idiopathic atrial fibrillation, observe (arrow) the 3 altered parameters: CEM (Ave), CEDP (standard deviation), and BDDP (standard deviation). To the right, in a patient with atrial fibrillation and chagasic cardiomyopathy, the 3 parameters studied are normal. 
nervous system, anisotropic conduction, and extrastimuli are also involved in triggering atrial fibrillation.

In the present study, 3 groups of individuals with paroxysmal or persistent atrial fibrillation were studied. Despite the similar female distribution among the groups, the analysis of age distribution showed that individuals with idiopathic paroxysmal or persistent atrial fibrillation are significantly older. This fact seems to reflect the epidemiological characteristics of the diseases in question.

Until the present time, no typical morphological alteration in atrial fibrillation has been reported. Fibrosis and fat infiltration, such as the fibrosis that occurs in atria in chronic atrial fibrillation, are alterations found in healthy hearts of elderly individuals. Occasionally, specific conditions like tumors, amyloidosis, or lipomatous hypertrophy may constitute arrhythmogenic histological substrates.

Atrial fibrillation has been the object of increasing interest for wider clinical research, due to the hemodynamic deterioration and the increase in morbidity and mortality that this arrhythmia may generate, with an important impairment in the quality of life and a significant increase in medical costs. Atrial fibrillation is almost always associated with a heart disease or clinical disorder; however, in up to $30 \%$ of cases, no evidence of structural cardiac disease exists ${ }^{9}$.

QRS-complex signal-averaged electrocardiography has been used in recent years to identify patients at risk for potentially fatal ventricular arrhythmias ${ }^{10}$. In 1991, Kelen et al ${ }^{11}$ proposed a new model to compensate for some disadvantages of the time-domain analysis and the methods used until then for frequency-domain analysis ${ }^{12}$. This technique includes the analysis of the QRS complex as a whole and not only the previously determined temporal part of frequency or amplitude.

Frequency-domain mapping is characterized by segmentation of the electrical signal at fixed intervals, decomposing it in elementary frequencies, through the use of rapid Fourier transform. The mark of the arrhythmogenic abnormality is theorized to be the frequent and abrupt change in the direction of the wave front activation, crossing areas of abnormal conduction, resulting in the presence of spectral turbulence ${ }^{13}$.

Based on P-wave spectral mapping, some authors investigated spectral turbulence as a marker of intraatrial conduction disorders ${ }^{13-15}$. In this context, P-wave signalaveraged electrocardiography proved to be a noninvasive, low-cost, easily performed and reproducible method; therefore, it has been widely used in the evaluation and stratification of the risk of atrial fibrillation ${ }^{16-27}$.

Due to the presence of clear cardiac structural changes, patients with atrial fibrillation associated with rheu- matic disease and other forms of heart disease would be expected to have more alterations in atrial electrical activation detected on P-wave signal-averaged electrocardiography. However, the electrophysiological alterations characterized by spectral turbulence in individuals with idiopathic paroxysmal or persistent atrial fibrillation indicate the existence of intraatrial conduction disorders as intense as or more intense than those observed in patients with paroxysmal or persistent atrial fibrillation and structural heart disease.

Previous studies have shown that frequent monomorphic atrial extrasystoles may trigger episodes of atrial fibrillation, and that ablation of this focus with a catheter may prevent future episodes ${ }^{28}$. This concept of focal atrial fibrillation may explain, in some patients, the recurring episodes of this arrhythmia despite the existence of a structurally normal heart. On the other hand, other studies in patients with paroxysmal or persistent atrial fibrillation have reported histological abnormalities of the atrial myocardium characterized by fibrosis and amyloid infiltration, both in the rheumatic and in the idiopathic forms of this arrhythmia ${ }^{29,30}$. However, no data exist in the literature allowing the comparison between the different atrial histologic patterns according to each etiology and the correlation with $\mathrm{P}$-wave electrocardiographic findings. A reasonable hypothesis to be considered, still lacking scientific confirmation, is that the cause of idiopathic paroxysmal or persistent atrial fibrillation is a primary alteration in the atrial myocardium similar to that found in rheumatic patients with fibrosis or deposition of amyloid substances, facilitating the formation of reentrant circuits. In this condition, idiopathic paroxysmal or persistent atrial fibrillation will show an electrophysiological pattern of atrial activation similar to that of rheumatic heart disease, in which these structural alterations are frequently found.

In the present study, we show that alterations in intraatrial electrical conduction are found with the same frequency or more frequently in individuals with idiopathic paroxysmal or persistent atrial fibrillation than in those with several heart diseases, suggesting the presence of a type of structural involvement of the atrial myocardium, which cannot be detected on conventional electrocardiography and echocardiography.

As limitations of this study, we can point out that the P-wave alignment used allows a direct comparison of the waveforms, with exclusion of ectopic beats. However, due to P-wave morphological characteristics represented by low voltage values and of the first derivative, as compared with those of the QRS complex, the error of alignment of waveforms may be high, generating less accurate analyses. More advanced systems of P-wave coherent averaging may be required to support our findings. 


\section{References}

1. Zipes DP. Specific arrhythmias: diagnosis and treatment. In: Braunwald E (Ed). Heart Disease. $5^{\text {th }}$ ed. Philadelphia: WB Saunders, 1997: 654-6.

2. Myerburg RJ, Kessler KM, Castellanos H. Recognition, clinical assessment and management of arrhytmias and conduction disturbances. In: Schlant RC, Alexander RW (Eds). The Heart. $8^{\text {th }}$ ed. New York: McGraw-Hill, 1994: 725-9.

3. Falk RH. Atrial fibrillation. In: Podrid PJ, Kowey PR (Eds). Cardiac Arrhythmia. $1^{\text {st }}$ ed. Maryland: Williams Wilkins, 1995: 803-27.

4. Werkö L. Atrial fibrillation: introduction. In: Olsson SB, Allessie MA, Campbell RWF (Eds). Atrial Fibrillation: Mechanisms and Therapeutic Strategies. $1^{\text {st }}$ ed. New York: Futura Publishing, 1994: 1-13.

5. Lévy S, Breithardt G, Campbell RWF, et al. Atrial fibrillation: current knowledge and recommendations for management. Eur Heart J 1998; 19: 1294-320.

6. Barbosa EC, Barbosa PRB, Ginefra P, Albanesi $\mathrm{F}^{\circ}$ FM. Frequency analysis of the signal averaged $E C G$ of the $P$ wave for identification of patients at risk of recurrence of idiopathic atrial fibrillation. J Am Coll Cardiol 1998; 31: 40C.

7. Ohe T, Matsuhisa M, Kamakura S, et al. Relation between the widening of the fragmented atrial activity zone and atrial fibrillation. Am J Cardiol 1983; 53: 1219-22.

8. Allesie MA, Konings K, Kichhof C. Mapping of atrial fibrillation. In: Olsson SB, Allessie MA, Campbell RWF (Eds). Atrial Fibrillation: Mechanisms and Therapeutic Strategies. $1^{\text {st }}$ ed. New York: Futura Publishing, 1994: 37-49.

9. Prystowsky EN, Benson DW, Fuster V, et al. Management of patients with atrial fibrillation. Circulation 1996; 93: 1262-77.

10. Simsom MB. Use of signals in the terminal QRS complex to identify patients with ventricular tachycardia after myocardial infarction. Circulation 1981; 64: 235-46.

11. Kelen GJ, Henkin R, Starr AM, CarefEB, Bloomfield D, El-SherifN. Spectral turbulence analysis of the signal averaged electrocardiogram and its predictive accuracy for inducible sustained monomorphic ventricular tachycardia. Am J Cardiol 1991; 67: 965-75.

12. Haberl R, Jilge G, Putter R, Steinbeck G. Spectral mapping of the electrocardiogram with fourier transform for identification of patients with sustained ventricular tachycardia and coronary artery disease. Eur Heart J 1989; 10: 316-22.

13. Barbosa EC, Barbosa PRB, Ginefra P, Albanesi Fo FM. Oeletrocardiograma de alta resolução no domínio da frequiência. utilização de técnicas estatísticas de correlação espectral para identificação de pacientes com taquicardia ventricular monomórfica sustentada. Arq Bras Cardiol 1998; 71: 595-9.

14. Barbosa EC, Barbosa PRB, Ginefra P, Albanesi $\mathrm{F}^{\circ} \mathrm{FM}$. The frequency analysis of the signal-averaged ecg of the $p$ wave is a good predictor of the efficacy of class iii antiarrhythmic drugs to maintain the sinus rhythm in patients with recurrent idiophatic atrial fibrillation. J Am Coll Cardiol 2000; 35(suppl A): 131A.

15. Stafford PJ, Vincent R. Spectrotemporal and spectral turbulence analysis of the signal averaged $\mathrm{p}$ wave in paroxysmal atrial fibrillation. J Electrocardiol 1997; 30:79-86.
16. Steinberg JS, Zelenkofske S, Wong SC, Gelernt M, Sciacca R, MenchavezE. Value of the P-wave signal averaged ECG for predicting atrial fibrillation after cardiac surgery. Circulation 1993; 88: 2618-22.

17. Frost L, Lund B, Pilegaard H, Christiansen EH. Re-evaluation of the role of pwave duration and morphology as predictors of atrial fibrillation and flutter after coronary artery bypass surgery. Eur Heart J 1996; 17: 1065-71.

18. Baciarello G, Bella R, Di Maio F, et al. Analysis of high resolution atrial activation: report on 403 cases. Am Heart J 1987; 113: 307-15.

19. Abe Y, Fukunami M, Yamada T, et al. Prediction of transition to chronic atrial fibrillation in patients with paroxysmal atrial fibrillation by signal averaged electrocardiography. Circulation 1997; 96: 2612-6.

20. Klein M, Evans SJ, Blumberg S, Cataldo L, Bodenheimer MM. Use of p-wave triggered P-wave signal-averaged electrocardiogram to predict atrial fibrillation after coronary bypass surgery. Am Heart J 1995; 129: 895-901.

21. Cecchi F, Montereggi A, Olivotto I, Marconi, Dolara A, Maron BJ. Risk of atrial fibrillation in patients with hypertrophic cardiomyopathy assessed by signal averaged $\mathrm{P}$ wave duration. Heart 1997; 78: 44-9.

22. Chang AC, Winkler JB, Fananapazir L. P wave signal averaging identifies hypertrophic cardiomyopathy patients with paroxysmal atrial fibrillation. J Am Coll Cardiol 1990; 12: 191

23. Murakami Y, Okamoto M, Nakayama R. Atrial late potencial by $P$ wave triggered signal averaged electrocardiogram and paroxysmal atrial fibrillation. Nippon Rionsho 1995; 53: 489-95.

24. Keane D, Stafford P, Baker S, Lewis S, Jackson G, VincentR. Signal averaged electrocardiography of the sinus and paced $\mathrm{P}$ wave in sinus node disease. Pacing Clin Electrophysiol 1995; 18: 1346-53.

25. Dimmer C, Jordaens L, Gorgov N, et al. Analysis of the P wave with signal averaging to assess the risk of atrial fibrillation after coronary artery bypass surgery. Cardiology 1998; 89: 19-24.

26. Garcia AA, Valdes CM, Vicente VT, Garcia RJ, PerezLF. The spectral analysis of the $P$ wave in patients with paroxysmal atrial fibrillation. Rev Esp Cardiol 1994; 47: 303-7.

27. Michelucci A, Padeletti L, Chelucci, et al. Influence of age, lead axis, frequency of arrhytmic episodes and atrial dimensions on $P$ wave triggered SAEGG in patients with lone atrial fibrillation. Pacing Clin Electrophysiol 1996; 19; 758-67.

28. Jais P, Haissaguerre M, Shah DC, et al. A focal source of atrial fibrillation treated by discrete radiofrequency ablation. Circulation 1997; 95: 572-6.

29. Frustaci A, Chimenti C, Bellocci F, Morgante E, Russo MA, Maseri A. Histological substrate of atrial biopsies in patients with lone atrial fibrillation. Circulation 1996; 96: 1180-4.

30. Bustos D, Perrenoud JJ, Michel JP, Grab B, McGee W. Postmortem diagnosis of cardiac amyloidosis in the aged. anatomoclinical correlation. Arch Mal Coeur Vaiss. 1993; 86: 315-9. 